\title{
Regard cosmopolite: réflexion sur le viol des femmes en Bosnie
}

\author{
France Théoret
}

\begin{abstract}
A Cosmopolitan Project
Reflections on the raping of women in Bosnia

The order of things is no longer the same when one knows of the widespread raping of Bosnian women. But misinformation has been an important weapon in this war. Why have feminist journalists, academics and writers failed to disseminate the facts? Fifty thousand women have already been raped; at this level, nothing is relative. Today, it is the responsibility offeminists to intervene and insist that these crimes be recognized. One can no longer be a feminist for oneself, for one's own enclave; feminist consciousness must become cosmopolitan.
\end{abstract}

L'avènement des femmes en politique appartient à notre époque, où elles ne sont pas assez nombreuses pour être influentes. Le féminisme, né d'une prise de position politique à la fin du $18^{\mathrm{e}}$ siècle avec Mary Wollstonecraft et Olympe de Gouges, relate des périodes d'émergence suivies de «backlash», comme on le dit aujourd'hui. L'histoire du féminisme n'est pas linéaire, son développement est discontinu. La connaissance que nous avons de son histoire doit réorienter nos perceptions de la réalité.

Maintenant les événements internationaux sollicitent notre attention et la présence des femmes en politique manque gravement. Je pense avec Hannah Arendt et d'autres philosophes contemporains que ce qui se passe ailleurs a des conséquences içi. Les nombreuses réflexions actuelles sur l'Holocauste doivent féconder notre vigilance. Il a été dit: plus jamais. Si l'histoire régresse dans la barbarie, on ne peut plus dire qu'on ne savait pas.

Nous possédons des informations, nous avons les moyens de savoir ce qui se passe à l'étranger. Un bon nombre de femmes sont des journalistes professionnelles, ont accès au pouvoir des médias. Faire connaître, provoquer la réflexion, approfondir un sujet d'intérêt concernant les femmes dans le monde est nécessaire si nous désirons maintenir une 
conscience féministe qui doit être ouverte à l'alterité. Il ne suffit plus d'être féministe pour soi-même, pour son groupe, pour une société particulière.

On ne peut plus rendre abstraites, conceptuelles des tragédies lointaines sous prétexte qu'elles ne touchent pas son groupe ou la société que nous habitons. Entre les idées et l'engagement personnel et collectif, il y a, dans une période de morosité, une telle distance qu'on ne s'engage plus et les prétextes des attitudes de repli sont diversifiés. Une vision cosmopolite est à développer, non pas uniquement en regard des idées générales. Il faut à coup sûr continuer d'enrichir la pensée. Mais il est nécessaire de connaître, de prendre position, la passivité et l'indifférence étant une complicité.

On n'a pas le droit de dire que les conflits mondiaux sont interchangeables. On n'a pas le droit de dire que les torts s'équivalent, que les responsabilités sont partagées. On ne peut pas fondre dans un même tout, victimes et bourreaux, pour s'en laver les mains, avoir une conscience tranquille, la soulager à moindres frais. On ne peut pas nier la réalité, agir par dénégation. Nous devons connaître ce qui se passe maintenant et refuser de se taire sur les crimes contre les femmes.

Le phénomène des viols commis en Bosnie contre les femmes musulmanes est sans précédent. Le fait a été réduit pour atténuer l'impact sur l'opinion publique, on l'a réduit à une incidence circonscrite, propre aux violences d'une guerre. La communauté politique et les médias ont banalisé l'importance des viols collectifs, suivis dans beaucoup de cas, d'assassinat sitôt le viol perpétré. Des viols ont été commis systématiquement et délibérément par les Serbes sur les femmes musulmanes. Ces viols collectifs faisaient partie d'une stratégie d'élimination et du nettoyage ethnique.

On veut banaliser l'ampleur de ces crimes et rendre le nombre de ses victimes insignifiant. Qui encore? Les politiciens et les médias.

Il y a eu également des femmes croates qui ont été violées mais c'est la première fois dans l'histoire que des ressortissantes d'un groupe religieux et ethnique sont désignées comme cibles de viols et d'assassinats.

Un rapport produit par l'organisation féministe croate « Tresnjevka » relate l'existence de camps de concentration-bordels. L'organisation en a identifié pas moins de seize. Les femmes entre dix et trente ans ont subi, chaque jour, entre quarante et cinquante assauts sexuels. Il s'en est suivi des "maladies vénériennes, des blessures internes, des privations de 
nourriture, des tortures et d'autres formes d'humiliation» (1). On n'en parle pas, c'est le secret le mieux gardé.

Les femmes en âge de procréer qui sont devenues enceintes, on été séquestrées, on les a empêché d'avorter en leur disant qu'elles devraient mettre au monde un enfant serbe. Celles qui ont pu sortir enceintes de cinq ou six mois n'ont pas pu avorter et plusieurs préféreraient tuer l'enfant à la naissance. Le viol est devenue une arme génétique.

Il s'agit d'un crime que même les nazis n'avaient pas expérimenté. Ces femmes sont condamnées à porter, à nourrir, à vivre au plus profond d'elles-mêmes avec ce qui les nie à jamais. Chacune des femmes individuellement vit les ravages du national-communisme.

Jean-Paul II, qui se distingue encore par son obscurantisme, a demandé aux femmes violées enceintes de garder l'enfant pour mesurer la force de l'horreur yougoslave.

Dans le rapport, une croate dont le nom n'est pas mentionné dit qu'elle " a écouté les femmes médecins et les victimes pendant des heures, a pris des notes, fait des enregistrements sur bandes et cassettes vidéos ». Elle a déclaré: «Le monde doit simplement savoir que c'est une guerre contre les femmes ».

De ces prisons-bordels, il y a celles qu'on a amenées jusqu'aux tranchées en première ligne pour satisfaire les guerriers.

Il y a les disparues, celles qu'on a assassinées, celles que les tchetniks ont entraînées hors le camps, pour les tuer après les viols.

On a violé des fillettes à partir de l'âge de six ans. Une pratique courante a été de violer en présence de membres de la famille : la mère, le père, la fille, le fils, le mari ou les grands-parents pour amplifier la destruction de la famille, dans le but d'humilier et de dégrader physiquement et psychiquement.

Des femmes ont été violées par des voisins, des enfants par leur instituteur, la cruauté touche le lien social et la vision du passé est détruite.

On a fait état de mutilation des organes sexuels, d'empalement, de sévices corporels de toutes sortes.

Le viol a été utilisé aussi comme moyen de terreur, pour chasser les habitants de leur lieu séculaire, pour effectuer le nettoyage ethnique $d^{\prime} u n$ village, d'une ville. Les viols se poursuivent, la guerre n'est pas terminée.

Il est impossible de réduire l'importance de ces viols et leurs conséquences. Dr. Zuhra Dizdarevic de Sarajevo, de la clinique gynécologique de l'hôpital Kosevo, affirme qu'une situation alarmante a 
commencé durant les mois de l'été 1992. Il y a eu mensuellement plus de 400 femmes victimes de viol qui ont subi un avortment dans cette seule clinique. Il existe d'autres cliniques. Un responsable auprès du gouvernement de la Bosnie, Smajo Ceklio mentionne le nombre de 50,000 femmes violées. Le Service de Santé en Bosnie indique 38,000 femmes violées dont 3,000 à Sarajevo. Le Service de Santé a enregistré 9,000 femmes enceintes suite aux viols et, à sa connaissance, 387 cas de décès survenus suite à la bestialité du viol. Le State Department affirme catégoriquement qu'il y a eu 30,000 femmes violées. Il nous faut connaître l'ampleur de ces crimes et faire connaître ce que nous savons. Après une querelle de chiffres, les médias ont tenté de nous faire croire à des incidents négligeables alors qu'il s'agit d'une vérité insoutenable.

Au début de 1993, le Conseil de sécurité a manifesté la volonté de créer un tribunal international pour juger des crimes de guerre. Une commission a adopté une résolution qui autorise la création de ce tribunal qui devrait juger les crimes contre l'humanité commis sur le territoire de l'ex-Yougoslavie. Des groupes et des personnes, dont Simone Veil, ont demandé que les viols perpétrés durant la guerre soient jugés au même titre que les autres catégories de crimes. Pour la première fois dans l'histoire, le viol serait reconnue commes les autres crimes de guerre.

Les femmes, individuellement et collectivement en tant que féministes, doivent intervenir pour que ces crimes soient reconnus comme tels dans le droit international.

Le tribunal a été instituté, les juges ont été choisis et assermentés.

Même théoriquement, le viol fera-t-il partie des catégories de crimes contre l'humanité ? Il ne le sera pas sans des prises de position de la part des femmes, sans leur volonté. Nos porte-paroles seront les journalistes, les femmes en politique et les juristes en droit international.

Il faut souligner le peu de poids des résolutions du Conseil de securité qui s'inscrivent rarement dans les faits. Dans l'énoncé des textes, tout est mitigé et relativisé. Lorsque quelques mesures d'action s'y trouvent, elles ne sont pas appliquées. Brièvement et à titre d'exemple, la livraison de l'aide humanitaire stipule que les soldats de l'ONU ont le droit de recourir à la force pour l'acheminer. La force n'a jamais été utilisée, pas même comme moyen de menace. Lorsque bloquée par les milices serbes, les soldats doivent quémander le droit de passage aux agresseurs qui sont les violeurs et les criminels. Lorsque les agresseurs autorisent le passage du convoi après plusieurs tentatives, ils prélèvent, de poste en poste de contrôle, une partie de l'aide. Les victimes reçoivent moins de 
la moitié de l'aide qui leur était destinée, avec parfois des semaines et des mois de retard. Un autre exemple serait celui des zones de securité qui ne sont pas toujours respectées.

Le tribunal étant mis sur pied, il est d'ores et déjà connu que son pouvoir est à l'avance réduit. Ce tribunal qui devra siéger à LaHaye pour juger des crimes commis sur le territoire de l'ex-Yougoslavie, n'a aucune autorité pour émettre des mandats d'amener, aller chercher les présumés coupables et les traduire en justice. S'ils sont condamnés, le tribunal ne peut ordonner leur incarcération. Les juges ne peuvent prononcer des arrêts de mort, ni juger par contumace, quels que soient la gravité et le nombre des crimes commis.

C'est à l'opinion mondiale et aux groupes de pression de voir à ce que le tribunal opère. C'est aux mouvements de femmes de voir à ce que le viol relève des crimes abjects commis contre l'humanité, qu'ils soient inscrits et reconnus dans le droit international, qu'ils figurent en tant que délits dans l'acte d'accusation.

Il nous faut dénoncer les crimes commis sur les femmes musulmanes et croates catholiques. Jusqu'içi il y a eu des actions humanitaires pour leur porter secours. Il y a eu beacuoup d'engagements à titre personnel comme Simone Veil, par exemple, pour dire les faits et les porter à notre connaissance. Mais il n'y a pas eu d'engagement collectif des féministes. On ne peut pas dire qu'on ne savait pas, on doit admettre que des dizaines de milliers de femmes violées, ce n'est pas une incidence négligeable dont on peut parler en quelques phrases dans un journal. Le fait est considérable et, de sa vérité, on ne peut parler en termes généraux, de façon idéologique avec des idées abstraites.

La conscience féministe doit être cosmopolite. Il faut dire ce qui se passe. Dans cette guerre d'agression, de massacre et de destruction par la Serbie et les Serbes de Croatie et de Bosnie, qualifiée, désignée faussement comme une guerre civile par les médias, il y a de la désinformation et de la propagande.

Rien n'est interchangeable au niveau des faits. On ne peut pas les banaliser ni les relativiser. Du viol des femmes, les politiciennes doivent parler, lesjournalistes doiventécrire. Les décisions qui seront prises relativement au tribunal international ne seront effectives que si les femmes agissent et veillent à ce que les intentions se traduisent dans les faits et que les violeurs soient jugés et condamnés.

Il y a du cynisme en politique et de la désinformation par des inexactitudes dans les médias. Le viol est un crime abjet, le refus d'en parler 
constitue une complicité avec les violeurs et ceux qui veulent ignorer la réalité. Car s'il y a bien une attitude répandue, c'est de repousser, dénier les faits qui ne font pas l'affaire, pour maintenir une fiction selon laquelle le devenir des ideées serait indépendant de la réalité. Il y a un confort intellectuel à ne pas tenir compte de la réalité qui arrange les assassins quels qu'ils soient. Ça s'appelle de l'anesthésie morale.

Maintenant, on ne peut plus continuer à être féministe pour soimême, pour son groupe, pour la société que nous habitons sans nous couper de la réalité du monde. Je m'étonne que des journalistes, des professeures, des écrivaines n'aient pas écrit sur la tragédie des femmes en Bosnie. Le féminisme ne s'est pas réclamé d'une pensée détachée de la réalité, il n'est past une théorie spéculative, conceptuelle et formelle, il n'est pas enfermé dans la déconstruction-contestation, le mode de pensée le plus constant en Occident. Il nous faut être cosmopolites, non pas dans la fiction et la représentation, mais en tenant compte des faits qui dérangent. La tragédie des femmes bosniaques déplace le centre des préoccupations du féminisme. L'ordre des choses n'est plus le même quand on sait.

\section{Ouvrages cités}

Le Livre noir de l'ex-Yougoslavie, Purification ethnique et crimes de guerre, éd. Arléa, Paris 1993.

Note: à paraître en décembre 1993 ou janvier 1994, L'Ex-Yougoslavie et le viol des femmes, éd. Eulina Carvalho, sous la direction d'Erika Hapfelbaum. 